\title{
Rhubarb and Reliability - A Jane Austen View of Systemic Lupus Erythematosus
}

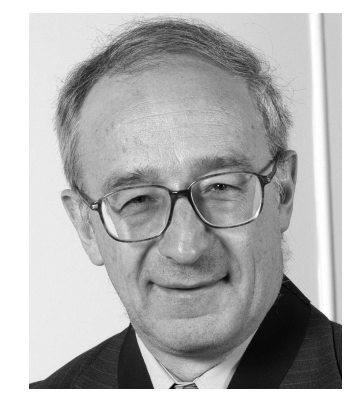

It is a truth universally acknowledged that a rheumatologist in possession of good sense draws comfort from confirming his or her impression about the degree of activity of a patient with systemic lupus erythematosus (SLE) by using a reliable disease activity index and appropriate laboratory investigation. From the mid-1950s to the mid-1980s, about 60 different disease activity indices were reported for SLE ${ }^{1}$. These indices were all global scores and none of them was ever shown to be reliable, validated, or sensitive to change. From the 1980s onward, however, considerable thought by several groups went into improving the quality of activity indices, with considerable effort made to demonstrate their validity, reliability, and sensitivity to change ${ }^{2}$. There has been a great debate as to whether as complex a disease as SLE can be captured by a simple global activity index. The global score has the attraction of simplicity and makes good sense if, for example, a new antibody test has been developed, e.g., an anti-rhubarb antibody that is claimed to be very effective at reflecting disease activity. It would be relatively easy to collect serum from patients with high global activity score and a similar set of samples from those with no or little disease activity, as measured by the global score system, and simply compare the anti-rhubarb antibody results. Equally, longitudinal studies using such a global activity index and antibody levels would help to validate the potential utility of the anti-rhubarb antibody as an objective biological marker.

However, the Achilles heel for the global score is the rigidity with which it is usually associated, i.e., if a clinical feature is deemed to be present, it gets points, if it is not present, it gets no points. The problem with this becomes evident when a patient presents with frank arthritis, for example, and is treated with the latest biologic or other therapy and a month later comes back to see their physician. Whether the patient claims to be $80 \%$ better, $80 \%$ worse, or the same as a month ago, he/she will receive the same number of points in a global score index. This is not only highly illogical, but in a clinical trial can be both misleading and unfortunate because significant improvements (or deterioration) caused by the drug will not be appreciated unless the symptom goes away completely.

It was precisely for this reason that the British Isles Lupus Assessment Group (BILAG) developed its own index ${ }^{3}-$ to get away from the black-and-white design of global score assessments. A key feature of BILAG is that the physician reviewing the patient is asked to determine whether individual clinical features are better, worse, the same, or a new/recurrent problem (within the last month). Based on these assessments, an individual activity score is recorded, from grade A (implying action, i.e., the patient is invariably going to require substantial amounts of steroids, immunosuppression, or anticoagulation) to grade $\mathrm{E}$ (implying that this particular feature has never ever been present). An updated and improved version of BILAG, BILAG-2004, is now widely available ${ }^{4}$.

It is, however, undoubtedly true that those interested in creating global activity indices in SLE, notably colleagues in Toronto developing the SLE Disease Activity Index $(\text { SLEDAI })^{5}$ score, and in Boston devising the Systemic Lupus Activity Measure (SLAM) score $^{6}$, have made substantial efforts to demonstrate the validity and reliability of their indices. The SLEDAI has also been updated and improved (SLEDAI-2K) ${ }^{7}$ and shown to be validated for a 30-day period in contrast to the original SLEDAI, which used a 10-day assessment window ${ }^{8}$. The BILAG and SLEDAI-2K instruments were incorporated together with a physician's global assessment score into a combined SLE Responder Index in a recent and successful trial of belumimab ${ }^{9}$.

In an interesting development, colleagues in Toronto developed and validated the SLEDAI-2K Responder Index $50(\mathrm{SRI})^{10}$. This index measures $\geq 50 \%$ improvement in disease activity, i.e., it gets away from the concept that patients simply have or do not have particular SLE features. In a report published in this issue of The Journal ${ }^{11}$, the development of a user-friendly Website to provide training

See Users' satisfaction of the SRI Website, page 34

Personal non-commercial use only. The Journal of Rheumatology Copyright (c) 2013. All rights reserved. 
(and examination) for the SRI is described. The evaluation of this online training "tool" provides a useful advance and "companion" for a more comprehensive BILAG and SLEDAI-2K training program available from the Lupus Foundation of America.

As useful as the SRI may be, it is inadequate in one respect: it does not, unfortunately, identify those patients whose clinical features present 30 days ago are now worse, and until this deficiency is remedied, its wide use in clinical trials seems unlikely. Of course, the mischievous thought does occur that such an addition might lead to the revised index being termed the BILAG version of SLEDAI! In fairness, the author hastens to confess a longstanding role in the development of the BILAG system. But of course, Jane Austen captured this thought rather better when she (nearly) wrote in Pride and Prejudice, "Let us hope for better things. Let us flatter ourselves that both BILAG and SLEDAI may survive!"

DAVID A. ISENBERG, MD, FRCP, FAMS, Professor, Centre for Rheumatology, UCL Division of Medicine, The Rayne Building, 4th Floor, Room 424, 5 University Street, London WC1E 6JF, England.

E-mail: d.isenberg@ucl.ac.uk

\section{REFERENCES}

1. Liang MH, Socher SA, Larson MG, Schur PH. Reliability and validity of six systems for the clinical assessment of disease activity in systemic lupus erythematosus. Arthritis Rheum 1989;32:1107-18.

2. Romero-Diaz J, Isenberg D, Ramsey-Goldman R. Measures of adult systemic lupus erythematosus: Updated version of British Isles Lupus Assessment Group (BILAG 2004), European Consensus Lupus Activity Measurements (ECLAM), Systemic Lupus Activity Measure, Revised (SLAM-R), Systemic Lupus Activity Questionnaire for Population Studies (SLAQ), Systemic Lupus Erythematosus Disease Activity Index 2000 (SLEDAI-2K), and Systemic Lupus International Collaborating Clinics/American College of Rheumatology Damage Index (SDI). Arthritis Care Res 2011;63 Suppl:S37-46.

3. Hay EM, Bacon PA, Gordon C, Isenberg DA, Maddison P, Snaith ML, et al. The BILAG index: A reliable and valid instrument for measuring clinical disease activity in systemic lupus erythematosus. Q J Med 1993;86:447-58.

4. Yee CS, Farewell V, Isenberg DA, Prabu A, Sokoll K, Teh LS, et al. Revised British Isles Lupus Assessment Group 2004 index: A reliable tool for assessment of systemic lupus erythematosus activity. Arthritis Rheum 2006;54:3300-5.

5. Bombardier C, Gladman DD, Urowitz MB, Caron D, Chang CH. Derivation of the SLEDAI. A disease activity index for lupus patients. The Committee on Prognosis Studies in SLE. Arthritis Rheum 1992;35:630-40.

6. Chang ER, Abrahamowicz M, Ferland D, Fortin PR. Organ manifestations influence differently the responsiveness of 2 lupus disease activity measures, according to patients' or physicians' evaluations of recent lupus activity. J Rheumatol 2002;29:2350-8.

7. Gladman DD, Ibanez D, Urowitz MB. Systemic Lupus Erythematosus Disease Activity Index 2000. J Rheumatol 2002;29:288-91.

8. Touma Z, Urowitz MB, Ibanez D, Gladman DD. SLEDAI-2K 10 days versus SLEDAI-2K 30 days in a longitudinal evaluation. Lupus 2011;20:67-70.

9. Furie R, Petri M, Zamani O, Cervera R, Walker DJ, Tegzova D, et al. A phase III, randomized, placebo- controlled study of belimumab, a monoclonal antibody that inhibits B lymphocyte stimulator, in patients with systemic lupus erythematosus. Arthritis Rheum 2011;63:3918-30.

10. Touma Z, Gladman DD, Ibanez D, Urowitz MB. Development and initial validation of the Systemic Lupus Erythematosus Disease Activity Index 2000 Responder Index 50. J Rheumatol 2011;38:275-84.

11. Touma Z, Gladman DD, MacKinnon A, Carette S, Abu-Shakra M, Askanase A, et al. Development and assessment of users' satisfaction of the Systemic Lupus Erythematosus Disease Activity Index 2000 Responder Index-50 (S2K RI-50) Website. J Rheumatol 2013;40:34-9.

J Rheumatol 2013;40:7-8; doi:10.3899/jrheum.121224 